Article

\title{
Mapping Platform Urbanism: Charting the Nuance of the Platform Pivot
}

\author{
Ashlin Lee ${ }^{1, *}$, Adrian Mackenzie ${ }^{2}$, Gavin J. D. Smith ${ }^{2}$ and Paul Box ${ }^{1}$ \\ ${ }^{1}$ Land and Water Environmental Information Group, CSIRO, Canberra, ACT 2601, Australia; E-Mails: ashlin.lee@csiro.au \\ (A.L.), paul.box@csiro.au (P.B.) \\ ${ }^{2}$ School of Sociology, Australian National University, Canberra, ACT 0200, Australia; E-Mails: adrian.mackenzie@anu.edu.au \\ (A.M.), gavin.smith@anu.edu.au (G.J.D.S.) \\ * Corresponding author
}

Submitted: 6 October 2019 | Accepted: 13 January 2020 | Published: 13 March 2020

\begin{abstract}
Urban planners are increasingly working with ideas around datafied cities, such as platform urbanism, to understand urban life and changes with technology. This article seeks to assist urban planners in these efforts by analysing and mapping the qualities of platform urbanism. Drawing on a dataset of approximately 100 examples that detail urban data practices, we trace some of the current tendencies that are shaping the nature and dynamics of platform urbanism. While we identify no unifying narrative or overarching pattern to our data, we interpret this as supporting Barns' (2019) notion of a pivot towards platforms. We argue this through exploring the interoperability between data sources and domains (vertical and horizontal integration), identifying elements of how platforms intermediate urban life through their growth in different sectors and the use of geolocation, and note the different artefacts that contribute to platform urbanism. We also note a concerning dynamic where city administration becomes 'locked in' to specific corporate products and interests, and thereby 'locked out' from alternatives. We discuss this in the context of social inclusion and what this means for urban planners, including the fragility of corporate platforms and what platforms urbanism means for social relationships in the city.
\end{abstract}

\section{Keywords}

data; data markets; inequality; Internet of Things; platform urbanism; smart cities; urban informatics; urban planning

Issue

This article is part of the issue "Urban Planning and the Smart City: Projects, Practices and Politics" edited by Andrew Karvonen (KTH Royal Institute of Technology, Sweden), Matthew Cook (Open University, UK) and Håvard Haarstad (University of Bergen, Norway).

(C) 2020 by the authors; licensee Cogitatio (Lisbon, Portugal). This article is licensed under a Creative Commons Attribution 4.0 International License (CC BY).

\section{Introduction}

Cities are increasingly being shaped by platforms, where patterns of consumption, socialising, and service provision are progressively being entwined with processes of datafication and digital platform technologies. While the idea of the 'smart city' has been widely associated with the intersection of data technologies and urban environments the unique affordances of platforms signal an evolution of the socio-technical relationship between citizens and cities. To anticipate and respond positively to these trends, urban planners must not only become fa- miliar with urban platforms, but understand their underlying dynamics, imaginaries, and practices. This article continues to develop ideas of platforms and urban life, specifically exploring 'platform urbanism' (see Barns, 2019; van der Graaf \& Ballon, 2019) and the sociotechnical artefacts and dynamics that create the conditions for this system of organisation to emerge. In doing so, we respond to Barns' call to explore the 'territories of platform intermediation,' investigating how the rise of platforms are changing urban socio-spatial practices and services, and consider what urban planners might do in response to these transformations (Barns, 2019, 
p. 7). We, therefore, move away from the notion of the smart city, and instead embrace the idea of platforms to explore the nuanced mixes of corporate homogeneity and socio-technical liveliness present in the contemporary city.

This article attempts to trace some of the current tendencies that are shaping the nature and dynamics of platform urbanism, using a landscape scan of approximately 100 urban data projects and their associated data practices. Our landscape scan dataset displayed no clear overarching narrative and is very contextual. We interpret this to offer support for Barns' notion of a pivot towards platform, and the re-orientation of different urban contexts towards platform technologies (Barns, 2019). We chart the interoperability between data sources and domains (vertical and horizontal integration), and highlight overlapping, and sometimes contradictory, processes of interoperability occurring between the diverse operators and users of these mediums. We also identify elements of how platforms intermediate urban life including in their growth in the public and private sector, and in the rising use of geolocation as a part of their operation. Finally, we note the three broad categories of artefacts that contribute to relationships and dynamics within platform urbanism: applications (apps), repositories, and platforms. Although there is no clear narrative or story to our data, there are patterns of data asymmetry occurring, as attempts by corporate actors to 'lock-in' their products while 'locking out' competition/alternatives mirror how the digital architecture of platforms leverages the openness or closedness of the system to generate value. We discuss this in the context of social inclusion and what this means for urban planners, including the fragility of corporate platforms and what the intermediation of platforms in urban life might look like.

\section{From Smart Cities to Platform Urbanism}

A sometimes ill-defined concept, the smart city broadly refers to how data technologies such as the Internet of Things (Zanella, Bui, Castellani, Vangelista, \& Zorzi, 2014), Big Data analytics (Kitchin, 2014), sensors (Hancke \& Hancke Jr., 2012), and pervasive Wi-Fi (Dohler et al., 2017) create what Kitchin (2014) calls 'the datafied city,' an urban space that is progressively constituted by processes of data capture and analysis. For Meijer and Bolívar (2016, pp. 396-397), datafication creates three (often siloed) visions of the smart city: (1) as instrumental applications of smart technology, (2) as places where datafication improves human capital and human outcomes, and ( 3 ) as a space for smart governance and more networked collaboration between different urban stakeholders. These visions are sponsored and promoted by corporations like CISCO and IBM, who use the smart city to propagate their corporate interests (Söderström, Paasche, \& Klauser, 2014) and, in some cases, lock cities into proprietary technical solutions (McNeill, 2015). This 'lock-in' dynamic holds a community or project to a specific vendor through the implementation of pathdependent technology, while also acting to 'lock-out' the same community from alternatives.

This vision is not totalising, however. Shelton, Zook, and Wiig (2015) show many purpose-built smart cities projects fail to develop as expected. Instead, they argue, more situated and empirically based accounts are needed to capture the heterogeneity of urban dynamics and technological innovation, and the often unanticipated and diverse practices that materialise as a consequence. At a technical level, Barns (2018) makes a similar point, contending that smart cities often stage a more varied proliferation of discrete data services and data assets, rather than universal or homogenised sociotechnical solutions. Although the individual nature and quality of services in smart cities vary, there is evidence of commonality. Data sharing practices and circulations are being increasingly brought together and organised through 'urban data platforms' (Barns, 2018). These platforms represent a new model of data-driven governance, characterised by different kinds of open data services-including city dashboards and data storesthat support new frameworks of urban management and public-private collaborations that capitalise on mass data flows from bodies, objects, and devices. This commonality is significant, because it foregrounds the growing influence of platforms on urban life, what Barns (2019) has described as the 'platform pivot,' as platforms emerge as a focal point for socio-technical and political economic modes of organisation in society. Platforms are not mere technical entities; they represent a unique socio-technical imaginary for enacting urban space and relations, and have significant implications for how urban planning is done.

'Platform' has a variety of definitions. The computational definition is perhaps the most common, describing an interoperable system comprising a set of stable core components or services, linked to an evolving set of peripheral or external components that have high variability (Baldwin \& Woodard, 2009), what we refer to as the core/periphery relationship. For instance, Facebook is a social media platform that provides a core set of social functions, but it has also created an environment for many other digital products (such as games and advertising content) that leverages off the core functions as part of a broader ecosystem of products. The core social media services of the Facebook platform are thus associated with a diverse set of peripheral applications that are constantly changing. This is facilitated through application programming interfaces (APIs), which allow third-party complementors access to the platform. Importantly, this access is asymmetrical; peripheral third parties who use APIs have little control over the stable core of the platform, while the core service often has significant power over third parties and the peripheral environment of apps that operate off the API. The Apple iOS ecosystem is a good example of this; it is a highly varied space for application development for Apple products, where app 
developers are often at the mercy of Apple for approval and distribution through the platform (Shilton \& Greene, 2019). The core/periphery relationship is, therefore, central to understanding platforms. For Bogost and Montfort (2009), variability defines the character of platforms because it allows original interactions that would otherwise be impossible; for example, the use of the Twitter API by a programmer to create a data visualisation that reveals new insights. Thus, the platform is not an intermediary carriage service without influence, but rather a mediator that actively shapes content and relationships linked to it (Latour, 2005). Gillespie (2010) notes how power is central to understanding the nature and function of platforms. Tensions arise between the agency of the user and more contentious value sets at the periphery, and the core service, which dictates the digital affordances of the platform (Helmond, 2015).

While often associated with social media, platforms have expanded into many social and economic domains, creating new tensions. For instance, Srnicek (2016) identifies how platforms have become central to most business models as companies realise how data can fuel growth and attempt to capture and utilise as much of this resource as they can. The data architecture of platforms provides an infrastructure that captures the data of all who interact on the platform, leveraging the core-periphery relationship to accumulate data and, therefore, to exercise power and accumulate profit (Srnicek, 2017). Network effects, or the power/value that comes from having more connections in a network, drive platforms to spread into more aspects of life and acquire more opportunities for data. The rise of Facebook, Amazon, Netflix, and Google, and the continuous digitisation of practices varying from healthcare (van Dijck \& Poell, 2016) to agriculture (Bronson \& Knezevic, 2016), are ample testaments to the power of this network effect, and to a business model obsessed with accumulating, commodifying, and monopolising the means of data production (Srnicek, 2017). The obsession with data has led to massive capital investments in infrastructures of data capture, raising valid concerns about platform surveillance and the manipulation of people's everyday data (Wood \& Mackinnon, 2019).

The rise of platform economies and capitalism is both a financial and ideological pursuit; it is driven by the technical architecture of platforms and the value of data accumulation, but also spearheaded through sociotechnical practices such as digital 'disruption.' Urban services like Uber, Airbnb, and Airtasker are all examples of this, disrupting existing service models in favour of platforms that decentralise service providers, but connect users and providers via platforms. While economically profitable for some, these transformations also present significant social implications, including insecure work and labour conditions (Pasquale, 2016). For example, in Barcelona, the arrival of Airbnb is viewed as damaging to the culture of the city, driving up rents and displacing young citizens from opportunities (Lambea Llop, 2017).
As van Dijck (2013) describes, a platform is often a constantly performed set of relationships that can set the agency and interests of one population (users, existing business holders, and communities, for example) against the platform's interests and operations (revenue generation, technical, social, or otherwise).

While discussion on smart cities has captured many of the entwinements between technology and urban life, a 'platform pivot' (Barns, 2019) is progressively occurring, as the socio-technical affordances and politicaleconomic ideologies of platforms come to dominate the character, feel, and organisation of contemporary cities. This is not necessarily a retreat from the notion of the smart city, but a recharacterisation of urban datafication that acknowledges the unique qualities and situations-but also growing potency-of platforms (Leszczynski, 2019). Van der Graaf and Ballon (2019) argue this uniqueness lies with how platforms conjoin commerce and community through the intersection of participatory technologies and practices, datafication, sharing environments, and cultures, and the multi-sided market that platforms create. Barns (2018) places platform urbanism in the context of urban data platforms, a range of data infrastructure that supports city governance and operation, including data warehouses, data marketplaces, and data showcases. The development of urban data platforms reflects the emergence of a new organisational logic concerned with managing and governing the city, with different urban data platforms embedding varied expectations and organisational content into civil, commercial, and citizen interactions. For Barns (2019) this makes the city less 'smart' as the affordances of platforms are tied to their often proprietary nature. The proprietary and highly commercial nature of platform urbanism is further highlighted by Caprotti and Liu (2020), who regard platform urbanism as linked to specific corporate, technological, and spatial geographies. While the role of corporations is often raised in the literature, Caprotti and Liu (2020) highlight the role of government in enabling platform urbanism; for example, Chinese state authorities are heavily invested in the development of urban data platforms. However, this may be related to the Chinese government's more centralised and authoritarian style of governance-its surveillance activities have benefited significantly from centralised data collection programs. Despite the variances illustrated above, there is a common interest in data, the intersection of data, and how urban life is increasingly animated and staged across platform types and geographies.

In line with the above discussion, we understand platform urbanism as the configuring of urban space around platform architectures that emphasise increased forms of data capture, programmability, automation, and thirdparty value generation (Helmond, 2015; Plantin, Lagoze, Edwards, \& Sandvig, 2018). For example, the urban data project proposed by Sidewalk Toronto involves a data infrastructure that allows urban data to be captured through a variety of sensors, with data amalgamated 
in a unified environment in which third-party innovations can occur (including artificial intelligence), creating value for the city (improved livability) and complementors (third parties who use the platform's data for innovation, services and products). Sidewalk Toronto is more than just a specific set of infrastructures or data collection tools that dataify and provide specific functions, but an overall structure that leverages a multitude of data through an integrated environment and marketplace. These structures facilitate the simultaneous 'opening up' and 'closing down' of diverse markets, services, and practices in quite sophisticated ways.

Our point of departure from this discussion is the need for deeper engagement with the specific sociospatial practices and services that are evolving in the wake of platform urbanism, and the different dynamics of interoperability between artefacts that operate alongside the logic of platforms. We noticed that many corporate actors are engaged in 'locking-in' urban spaces and communities to their platform products, while 'locking out' the possibility of alternatives. Unlike smart cities projects, platform urbanism amalgamates discrete urban data practices in both a centralised and decentralised fashion, so that various forms of urban activity (historical, actual, and speculative) can be better known and coordinated through a centralised point-the platform. In doing this, the platform simultaneously affords differential levels of openness, access, and service-depending on who or what is using them and what access rights and privileges they are granted. This brings service providers, users, and third-party complementors together in quite asymmetrical ways. It permits a fixed core to interact with a variable periphery linked together via interoperable data relationships. Our contribution to understand- ing platform urbanism is to situate and illustrate this dynamic in the context of existing data practices in the city, responding to Barn's call to explore the 'territories of platform intermediation,' and the nuances of how platforms shape urban space and living (Barns, 2019, p. 7)

This analysis becomes important, as models of platform urbanism progressively cement themselves at the core of urban life. Plantin et al. (2018) observed that platforms are increasingly melding withand transforming-existing urban infrastructure (see Table 1 for the qualities of platforms and infrastructure), revitalising the configuration of key urban services. This entails the platformisation of infrastructures, where public utilities are splintered into private services that are underpinned and controlled by private interests, and the infrastructuralisation of platforms, which give platforms the stable characteristics of-and appearance of being-public infrastructure. For infrastructuralised platforms, this includes responsibilities for providing longterm, standardised, scaled, and fundable services to large bodies of users, while also existing as an architecturally dispersed and capital-driven enterprise. While not exploring smart cities directly, Plantin et al.'s (2018) observation is evident in projects like Sidewalk Toronto, where Alphabet-owned Sidewalk Labs will provide the infrastructure for this urban space through the strategic deployment of its own private and for-profit technology, generating concerns around privacy, security, and in/equitability (Cecco, 2019).

As these architectures become more prevalent, and integrate more sources of data and human activity into their enclosures, critical questions must be asked to address platform urbanism and the cultures of capture, programmability, modulation, and value-generation its in-

Table 1. Infrastructure and platform properties.

\begin{tabular}{|c|c|c|}
\hline & Infrastructure & Platform \\
\hline Architecture & $\begin{array}{l}\text { Heterogenous systems and networks } \\
\text { connected via sociotechnical gateways }\end{array}$ & $\begin{array}{l}\text { Programmable, stable core systems; modular, } \\
\text { variable, complementary components }\end{array}$ \\
\hline Relation between components & Interoperable through standards & Programmability within affordances; APIs \\
\hline Market structures & $\begin{array}{l}\text { Administratively-regulated in public } \\
\text { interest; sometime private or public } \\
\text { monopoly }\end{array}$ & $\begin{array}{l}\text { Private competitive, sometimes regulated via } \\
\text { antitrust and intellectual property }\end{array}$ \\
\hline Focal interest & Public value; essential services & Private profit; user benefit \\
\hline Standardisation & Negotiated or de facto & Unilaterally imposed by platforms \\
\hline Temporality & Long-term sustainability; reliability & $\begin{array}{l}\text { Frequent updating for competitive } \\
\text { environment }\end{array}$ \\
\hline Scale & $\begin{array}{l}\text { Large to very large; ubiquitous, widely } \\
\text { accessible }\end{array}$ & $\begin{array}{l}\text { Small to very large; may grow to become } \\
\text { ubiquitous }\end{array}$ \\
\hline Funding & $\begin{array}{l}\text { Government; subscription; lifeline } \\
\text { services for indigent customers; } \\
\text { pay-per-use (e.g., tickets) }\end{array}$ & $\begin{array}{l}\text { Platform purchase (device), subscription } \\
\text { (online), pay-per-use (e.g., TV shows); } \\
\text { advertising 'Opt in,' for example; choosing } \\
\text { one platform instead of another; creating } \\
\text { mashups }\end{array}$ \\
\hline
\end{tabular}

Source: Reproduced from Plantin et al. (2018, p. 299). 
termediations facilitate. The sheer variability and scale of platforms, and the complexity of their social and technical arrangements, however, make any attempt to precisely and coherently summarise core facets challenging. While interoperability is a goal of many platforms, the social scripting and situatedness of technology means the operating reality of these entities is governed by contextual factors and circumstances, such as the technical components and operating cultures of the systems (Latour, 1996). Further, platforms are often connected to other platforms, forming what van Dijck, Poell, and de Waal (2018) call 'platform ecosystems,' which are heterogeneous assemblages of different platforms. Therefore, the first step to understanding the nuance of platform urbanism requires a means of categorising and mapping the variation in platforms themselves, and the underlying dynamics and processes existing between the different actors and arrangements in question. It is to this task that we now turn.

\section{A Reference Dataset for Platform Urbanism}

As part of a broader project on smart cities and equity in data economies and urban data markets, we conducted a 'landscape scan' of smart cities projects and initiatives (see the supplementary file for a full breakdown of examples scanned). Landscape scans are commonly used to strategically review programs or entities that exist within a specific context, for the purposes of business intelli- gence. This process is not meant to conduct deep, theoretical analysis of specific examples, but instead to establish the general trends and insights that can inform future practice. Three criteria were used to sample examples for the urban data market datasets:

1. Examples must be urban- or city-based;

2. Examples must involve data as a primary component;

3. Examples should attempt to share, circulate or otherwise mobilise data.

Using a snowball sampling approach (where each example guided us to find other relevant examples), we gathered relevant examples of initiatives, services, policies, projects and products explicitly labelled as 'smart city/cities.' We also included significant platforms, applications, and technologies that contribute to the realisation of smart city vision based on the inclusion criteria. We aimed for some global diversity, variations in scale and social setting, modest historical depth, and a mixture of the exotic and mundane.

The resulting dataset (see the Supplementary File), characterised by great heterogeneity, was then mapped against a set of general assessment criteria (see Table 2) synthesised from several different sources. Technical categories-such as the hardware and software environment-were drawn from the Reference Model for Open Distributed Processing (RM-ODP; Box \&

Table 2. Assessment criteria.

\begin{tabular}{|c|c|c|c|}
\hline $\begin{array}{l}\text { Architectural } \\
\text { Viewpoint }\end{array}$ & Dimension & Element & $\begin{array}{l}\text { Source } \\
\text { (adopt/adapt/invent) }\end{array}$ \\
\hline \multirow[t]{3}{*}{ Social } & Social & $\begin{array}{l}\text { Motivations for providing data; data } \\
\text { providing users relationship with } \\
\text { platform and its operators; scale and } \\
\text { extent of providers and users }\end{array}$ & Invented \\
\hline & Institutional & $\begin{array}{l}\text { Governance; stakeholder engagement; } \\
\text { ownership (drivers of the initiative) }\end{array}$ & $\begin{array}{l}\text { Adapted from Schreieck } \\
\text { et al. (2016) and Lee et al. (2018) }\end{array}$ \\
\hline & Economic & $\begin{array}{l}\text { Business model; incentives for } \\
\text { engaging with the platform sharing } \\
\text { data }\end{array}$ & $\begin{array}{l}\text { Adapted from Plantin et al. } \\
\text { (2018), Langley and } \\
\text { Leyshon (2017), and Lee } \\
\text { et al. (2018) }\end{array}$ \\
\hline \multirow[t]{2}{*}{ Technical } & Core and periphery & $\begin{array}{l}\text { Core components; peripheral } \\
\text { (application components); } \\
\text { functions provided by each }\end{array}$ & Adopted Lee et al. (2018) \\
\hline & Channels for data capture & Sensors & Adopted Lee et al. (2018) \\
\hline \multirow[t]{4}{*}{ Informational } & Sources of data & Where does the data come from & Invented \\
\hline & Theme of data & $\begin{array}{l}\text { Smart cities domains addressed } \\
\text { by the case study }\end{array}$ & $\begin{array}{l}\text { Adapted from loT and smart } \\
\text { cities literature }\end{array}$ \\
\hline & $\begin{array}{l}\text { Scale and scope of data } \\
\text { (and users) }\end{array}$ & Global, national, city, subcity & Invented \\
\hline & $\begin{array}{l}\text { Data as primary value } \\
\text { exchange or bi-product }\end{array}$ & & $\begin{array}{l}\text { Adapted from Schreieck } \\
\text { et al. (2016) and Lee et al. (2018) }\end{array}$ \\
\hline
\end{tabular}


Lemon, 2015). This model proposes five viewpoints in the management of distributed information systems: enterprise, information, computational, engineering, and technology. These viewpoints were collapsed into three architectural perspectives: social (enterprise viewpoint), informational (information viewpoint) and technological (computational, engineering, and technology viewpoints). Building on the framework from Box and Lemon (2015) the social perspective was expanded to include social (cultural and social practice aspects, including values, norms, and ethics), institutional (governance, decision structures and rights, and other institutional elements), and economic (incentives, value drivers, business model, etc.) dimensions. Given our interest in how data markets and platforms are configured, we also drew on recent interdisciplinary literature on platforms and data markets to analytically develop these categories (see Langley \& Leyshon, 2017; Lee, Zhu, \& Jeffery, 2018; Plantin et al., 2018; Schreieck, Wiesche, \& Krcmar, 2016 ). Results were synthesised using a design thinking methodology, as part of broader project deliverables such as apps and reports that are outside the scope of this article.

Analysis of the landscape scan dataset focused on counting and cross-tabulating examples. We used these counts to augment and adjust the dataset. If a particular count was low (for instance, examples in South East Asia or health-domain apps), we searched for more. If a particular count was too high, we reflected on the categories and sometimes refined them (by either adding new categories or perhaps new forms of classification). The process of creating such a reference dataset was potentially open-ended. Analytically, we regard this counting and tabulation of examples (see Tables 3 to 8 ) as ways of grounding the dominant narratives or representations of the somewhat free-floating concept of the smart city. Including more examples in understanding the smart city, especially mundane or low-profile examples, diversifies the concept. The counts and tabulations of examples inevitably flatten and reduce their lived complexities. However, the numbers can also deflect the insistent bias of power-laden conventional narratives.

There are limitations to this approach. Snowball sampling of public materials limited the scope and depth of examples. Publicly available information does not always capture the contexts of examples and might therefore be biased. Different team members coded the landscape scan; they may have potentially used different frameworks of assessment and understanding. Our criteria for classification, even at basic levels such as geographical location or social sector, were often difficult to apply and we struggled to consistently categorise what we were seeing despite multiple iterations through our 100 examples. We acknowledge these limitations vis a vis more indepth methods such as ethnography or even discourse analysis, but believe it still has merit. If nothing else, it presents the breadth and diversity of smart cities initiatives and their relationship to the notions of data markets. We suggest that some of the difficulties in sampling and analysing smart cities as processes of social transformation also derive from the shape-shifting practices of platform urbanism. As sociologist Howard Becker writes, 'phenomena seldom have all the attributes required for them to be, unambiguously, members of a class defined by multiple criteria' (Becker, 2008, p. 177).

\section{4. 'The Pivot': From Smart Cities to Platform Urbanism}

The landscape scan of smart cities shows no overall trend in the tabulated data, despite our efforts to find one. This null finding is not without interest. The collection of activities subsumed by the smart city, and the shifting composition of these activities, can be understood as an ongoing 'pivot' - a change of plans triggered by altered circumstances. This is first identified by Barns (2019), who suggests smart cities have pivoted using platform technologies, into what is previously described as platform urbanism. While identifying this general trend, the impacts and qualities of this pivot are unclear (Barns, 2019). Our results support Barns' (2019) theoretical observation and contribute towards elucidating the qualities of this pivot. We observed a pivot to platform urbanism in the dynamics of vertical and horizontal integration processes (defined in the following sections), and the amalgamation over time of urban data practice into the core-periphery relationships typical of platform configurations. Patterns of differential access and the lock-in/lock-out issue were also observed. But this is not a well-structured architectural transformation. Notwithstanding the many visions of integration we encountered, the landscape scan points to plurality, overlaps, provisional compromises and temporary fixes, and a constantly receding horizon of promise.

The amorphous character of the smart city is illustrated by geographical distribution of examples in the dataset (Table 3). Several spikes appear in the geography of the landscape scan. Many examples appear in a specific city (Kansas Smart City, Switching on Darwin, Sounds of New York City, Sydney Coordinated Adaptive Transport System), and 70\% of examples were associated with specific cities or were nationally scoped platform or app offerings from vendors (see Table 3). The remaining $30 \%$ of examples have a global scope and are not

Table 3. Geographical distribution of examples in the landscape scan.

\begin{tabular}{lc}
\hline Geography & Count \\
\hline Global & 34 \\
Multiple cities & 25 \\
Single city & 22 \\
National & 18 \\
Region & 3 \\
Neighbourhood & 3 \\
Dwelling & 3 \\
Unknown & 1 \\
\hline
\end{tabular}


Table 4. Vertical integration.

\begin{tabular}{llcl}
\hline & Verticality of smart cities technology & Count & Examples \\
\hline A & Platform only & 29 & ATandT Data Flow, IBM Watson, Microsoft \\
B & Sensors + Platform & 54 & CISCO Kinetic, Nokia Impact \\
C & Physical Infrastructure + Sensors + Platform & 16 & Bosch, Huawei, Microsoft, Telstra \\
D & Physical Infrastructure + Sensors & 4 & Smart fridges, air-conditioning \\
E & Sensor only & 2 & Analog Devices, Siemens Mindsphere, Philips \\
F & Infrastructure & 2 & Not considered, i.e., not 'smart' \\
G & Unknown/unclear & 2 & \\
\hline
\end{tabular}

tied to any particular city or local geography. These often more recent examples represent smart city idealisations and models of multinational technology vendors, or other service offerings that could be used anywhere. Examples range from Uber, to Pokémon GO to the Huawei City Platform.

\subsection{The Spaces of Interoperability: Vertical and Horizontal Integration}

Given the varying scale of examples, it is not surprising that we observed interoperability to be a key element of platforms. Without institutional, technical, and informational interoperability, a platform cannot generate the network effects around data that create value, or facilitate interactions between diverse sets of social and economic actors. We observed two kinds of interoperability-horizontal and vertical integrationwithin urban spaces that contribute to this. Vertical and horizontal integration refers to the ways in which information systems become more interoperable and interconnected in form and practice. Vertical integration refers to the coupling of socio-technical elements across the layers of physical and digital infrastructure that enable the capture, storage, and exploitation of data. Platform architectures have evolved 'stacks' that arrange and connect layers of technical elements. Our analysis shows a tendency towards greater vertical integration, replacing localised interfaces and targeted data sources with mass data collection flowing through platforms. We identified three categories of interfaces relevant to vertical integration: sensors (air, pollution, and water quality, for instance), physical infrastructure (such as smart lighting systems or roads), and platforms (data amalgamation and analysis services). As Table 4 shows, most smart cities examples feature some kind of interface between sensors and platforms, explicitly pairing urban data sensing with further analysis and operation by third parties, often through various types of automation.

The scope of data capture is also growing through horizontal integration, as Table 5 shows, with multiple fields of data associated to these platforms. Examples were assessed against all six domains to explore horizontal integration, resulting in multiple examples being coded to each criterion. A grand total is therefore unhelpful here. Horizontal integration refers to the expansion of data capture into different domains of human activity, and the integration of data from different sources and activities into platforms. Table 5 lists the specific scopes and domains of data collection we observed. Many smart cities examples no longer address a specific problem with specific data. Rather, they gather and accumulate data across domains, to anticipate and capitalise on diverse problems and possibilities yet to emerge. This shift in logic expands the remit of the smart city, in line with what Boyd and Crawford (2012) describe as one of the underlying mythologies of Big Data: that collecting all data ( $\mathrm{N}=$ all) is desirable-necessary even. This is evident in the increasing practice of collecting geolocation data, with Table 7 indicating most surveyed platforms use geolocation tracking as a default feature of their operation.

Table 5. Horizontal integration.

\begin{tabular}{lc}
\hline Data scope & Count \\
\hline Economy & 28 \\
Environment and energy & 28 \\
Government and education & 30 \\
Living and health & 43 \\
Safety and security & 24 \\
Mobility & 51 \\
\hline
\end{tabular}

The combination of these interfaces and data sources generates the pivot dynamics central to platform urbanism. The strata of urbanised platforms multiply and spread as smart cities pivot towards an ideal of agile entrepreneurial governance animated by metrics, experiments, models, dashboards, and continuous redeployment (Coletta \& Kitchin, 2017).

\subsection{Territories of Intermediation}

The use of platforms attests to steady growth in the markets for smart city products. This tends to creat the lock-in/lock-out dynamic. The platforms we observed to be growing in the domain of smart cities are primarily products created by corporate actors (Table 6). The earlier pre-eminence of governments and communities active in the smart cities domain subsides and, in recent years, private sector entities were the most dominant. Given that public sector entities often require the support of private vendors to initiate and deliver platform 
projects, we believe that many recent public projects also have a private sector dimension. As discussed earlier, smart cities implementors are incentivised to use proprietary technology to bolt clients to their products. For example, Huawei (2019) provides a 'holistic set of [smart city] ICT solutions featuring a "cloud-pipe-device" synergy.' This connects multiple kinds of devices (personal smartphones, sensor networks, smart infrastructure) that collect a multiplicity of data, displaying the vertical and horizontal integration described previously. It also functions to lock urban data and technologies into this environment by creating technological momentum (Hughes, 1994) that keeps the city beholden to the platform. This includes the significant capital cost of setup and installation - which, as McNeill (2015) noted, is sometimes waived to surreptitiously compel cities to continue using the platform due to the high initial costand the ongoing costs of having appropriately trained staff and expertise to operate the system. This prevents alternative vendors from engaging in this entity, creating the aforementioned lock-in/lock-out dynamics.

Table 6. Sectoral distribution.

\begin{tabular}{lc}
\hline Sector & Count \\
\hline Private sector/technology product & 52 \\
Government project & 26 \\
Civil society & 26 \\
\hline
\end{tabular}

Table 7. Use of geolocation.

\begin{tabular}{lc}
\hline Use of geolocation & Count \\
\hline Yes & 59 \\
Not Sure & 45 \\
No & 5 \\
\hline
\end{tabular}

\subsection{Artefacts of Platform Urbanism}

The dynamics of platform urbanism are created through a variety of services, products, and technologies-what might be thought of as the artefacts of platform urbanism. Combinations and relationships between and through artefacts (such as the interoperabilities previously discussed) allow effects like the lock-in/out dynamic to occur. For example, platforms such as IBM's Intelligent Operations Center for Smarter Cities can lock cities into centralised, and often privately operated, platforms. In doing so it can decontextualize data from urban environments and open data up for use by third parties, creating different kinds of products and services that affect urban life.

We identified three broad kinds of artefacts that contribute towards the platform pivot: applications (apps), repositories, and platforms (Table 8 ). Each artefact is a general category that can be used to understand the underlying nature of the examples we explored. Our classification of these examples derives from an interpretation of which artefact represents each example in our dataset.

Apps offer the platform environment of smartphones, such as the Apple iPhone or Google Android, a foothold on urban life. Although literature on smart cities has often focused on distributed hardware and software installations, we believe apps are important because of the distributed data-driven contribution they make to urban living. Apps carry the mundane tracking, capture, measurement, and communication operations that precede and underpin much platform urbanism by capturing metrics on the movement patterns of citizens to the metadata of communication in the city, contributing to liveability and the scripting of social relations. They prepare the foundation for platforms. For instance, from a pedestrian's perspective, apps superimpose data-driven interactions with a cyber-physical urban realm, such as through forms of recommendation (such as FourSquare) or site-specific entertainment and localised social interactions (like Pokémon GO or Grindr). Data generated through app interactions becomes a source of direct or indirect value at the platform level; for example, transport-as-service platforms like Uber generate value from creating a marketplace through the apps that connects third parties and enables new value to be generated from these transactions. The data from these exchanges can also be used as a source of value for the parent company. As a form of vertical integration, these apps unite the sensor and interface affordances of devices with decentralised software platforms that create geolocationally relevant and unique experiences for users, and provide this data to parent companies and third parties who add/derive value to/from it, matching characteristics of platform urbanism.

Table 8. Artefacts in platform urbanism.

\begin{tabular}{lc}
\hline Artefact & Count \\
\hline App & 32 \\
Repository & 31 \\
Platform & 28 \\
Other & 18 \\
\hline
\end{tabular}

Repositories are organisationally grounded centres of urban data storage and access, where data is centralised to enable decentralised value generation. According to Barns (2018), they are portals for machine-readable government data that seek to create innovation and transparency through providing centralised, but also deinstitutionalised and democratised, access to city data. Using standardised metadata, repositories enable different kinds of data to be stored and accessed thus enabling horizontal integration and potentially leveraging developments in vertical integration. Third parties are invited to create value from data collected about urban life and stored on repositories by downloading and using it, as repositories typically do not provide analy- 
sis functionality. Third parties, therefore, decontextualize the data away from its original context, creating different kinds of products and services as a result. This process is not inherently exploitative; for instance, the Chicago Data Collaborative is a repository of criminal justice data, adopting a cooperative model amongst members to store data for social justice causes.

Platforms are initiatives consistent with an earlier definition of platforms and their variable core/peripheral relationships. Large smart cities projects, such as Sidewalk Labs Toronto, adhere to this definition, providing a full socio-technical architecture that captures, stores, and analyses data, creating value for individuals and consumers. Platforms are more sophisticated multistakeholder or multisided entities, with the ability to collate, aggregate, integrate, and use multiple streams of data simultaneously. These projects are generally the domain of large technology companies, such as CISCO and IBM, due to development and deployment costs. They also reflect corporate interests and understandings of value, rather than community or ground-up visions. Their interfaces with city governance are uncertain and still taking shape.

While we have sought to highlight platform urbanism as the salient trend, the close integration of apps and repositories into the lived experiences of users takes platform urbanism into more socially situated spaces. Apps and repositories territorialise at the street and organisational level, respectively, evoking different data flows to create a varied and lively data ecosystem that, while coherent with the platform pivot is also nuanced.

\section{Discussion}

Platform urbanism, like the smart city before it, will likely be associated with a utopian promise of greater emancipation through data-driven insights and economies. While potentially true, it is important to not ignore the challenges and contradictions revealed by the nuances of the platform pivot. Two major, interrelated issues stand out from our analysis: (1) the agility and fragility of the platform pivot, and (2) the quality of intermediation (or the nature of socio-technical relationships under platform urbanism).

The idea of the pivot comes from business strategy and management literature, which emphasises the need for firms to flexibly re-orientate themselves in the face of market/user/customer change (Trimi \& Berbegal, 2012), an increasingly popular trend that dovetails with technology-centric management methodologies such as 'lean' and 'agile' (Bosch, Olsson, Bjork, \& Ljungblad, 2013). Our data supports the existence of a pivot, as the geographical distribution (Table 7), and vertical and horizontal integration metrics (Table 4 and Table 5) illustrate the movement towards platform systems across a variety of spatial areas and practice domains. The identification of the dual lock-in/lock-out dynamic of these platforms, however, makes us question whether this pivot is agile or is it more of a fragile entity? Horizontal and vertical integration indicate large volumes of data are available across multiple sectors. This suggests an ample volume of data for platforms to use and facilitate the platform pivot. If there is so much data being produced across so many sectors, why lock down communities? Locking down users and communities to platform products suggests, however, that solutions being provided by corporate technology providers are far more fragile than providers would have us believe. Despite the abundance and spread of data, there may be underlying issues making platforms vulnerable. We believe this may lie with a failure to understand the heterogeneity of urban life. Interoperability is vital to platforms because it ensures commensuration, allowing differing things to connect across contexts. Platforms use technical systems like APIs to allow different technologies to integrate, but it does not imply that social contexts will interoperate. The diversity of artefacts and contexts in which they operate (as identified above) means that for urban platforms to be realised, interoperability must be maintained against constantly active technical and social spaces-some of which may resist or disagree with the directions of corporate platform urbanism. The lock-in/lock-out dynamic is, therefore, a way of forcing interoperability to occur at a technical level, because the social reality of the city is more diverse than corporatised platform pivots expect. Furthermore, the idea of pivots and organisational agility don't fully capture the obduracy of some urban sociotechnical elements. This may include institutions, persistent socio-demographic deficits like poverty, or the physical environment. A pivot cannot occur without a fixed point; urban planners should be wary of fetishizing change, and missing those anchors that hold a platform together.

Building on the diversity of platform urbanism, and reflecting on the needs of urban planners to address the challenges of the city, the second issue we identify relates to the quality of socio-technical intermediation occurring in the city, and how urban planners might respond to this. What we refer to here is the nature of relationships occurring as a feature of platform urbanism, and that planners should be mindful of these relationships in how they understand urban issues. The diversity of artefacts, contexts, data sources, and practices identified suggests that the quality of intermediation a platform provides is more variable than suggested by private sector vendors. For example, projects such as the Common Sense program (Aoki et al., 2008) the Amsterdam Smart Citizens Lab (Jiang et al., 2016), and the Connected Sustainable Cities project (Gabrys, 2014), are examples of citizens using environmental sensors that are vertically integrated with a platform to create new kinds of social value, including contributing to better environmental outcomes and new practices of community informed urban governance. Many smart city vendors are private sector entities, whose vision of private profit contributes to the lock-in/lock-out charac- 
ter of platform urbanism that we observed. While platform capitalism (Srnicek, 2017) has configured many platforms to target new 'domains of circulation' (Langley \& Leyshon, 2017), there is potential for platforms to create new kinds of social and community value that urban planners could use to achieve social justice outcomes. Urban planners should therefore not accept homogenised visions and articulations of platform urbanism, as these ignore the social and technical diversity at play. As our results indicate, the reality of the smart city is one defined by considerable diversity and flux. Urban planners should thus consider the socio-technical dynamics inherent to relationships in the city, and how these can be used for making better design decisions and social policy formulations, rather than accepting any pre-existing template of the smart city that is driven predominantly by economic imperatives. For example, interoperability between diverse sets of artefacts suggests not only that data might be aggregated up into centralised platform architectures, but with the right configuration may also be pushed back down to users as civic resources. Studies of mobile technology users have demonstrated the incredible scope of apps and smartphone to create communities (Goggin \& Hjorth, 2014), even amongst the most disadvantaged groups in society, such as refugees (Gillespie, Osseiran, \& Cheesman, 2018). This illustrates how it is possible to interoperate diverse socio-spatial communities and practices while recognising the uniqueness of contexts. This may occur through opening up platform architectures socially and technically. Just as good urban planning co-design developments with communities, the same can-and should-be done with platforms. The APIs and technical architectures of platforms can also be configured to grant greater openness to those involved in the periphery, allowing them a greater voice in the form of platform urbanism being created. This embraces the diverse demographic and infrastructural constitution of smart cities despite powerful institutional actors and actions.

Building on this and our data, we also suggest that part of the quality of intermediation occurring under platform urbanism is one that is incoherent. This is a reality that urban planners must acknowledge and embrace, rather than resist or deny. While noting the dynamics of interoperability, we observed no overarching story or narrative that can easily capture all the relationships and dynamics of platform urbanism. We acknowledge that our approach is distinctly limited, and we may not be capturing enough to make this judgement, but it could also be that easily fitting narratives are not present in contemporary datafied cities. While there are sociotechnical hegemonies of platform and surveillance capitalism (Srnicek, 2017; Zuboff, 2015), neo-liberalism and political-economic uncertainty (Harvey, 2007), and stark social divisions, perhaps the reality of modern cities is one of incoherency, as both social groups and individuals attempt to make sense of socio-technical change that far outstrips understanding. For planners this means not at- tempting to impose singular understandings of urban life, and instead committing to a richer, more pluralised and deeply contextual view of urban relationships. Having an awareness of coherency is also not an excuse to divest from strategic and critical thinking. Planners should continue to be wary of how platform urbanism might reinforce disparities based on demographic characteristics such as income and geolocation (Brannon, 2017; GaldonClavell, 2013), and the continuing concerns about how data are collected, stored, used, and shared in the provision of services (Elmaghraby \& Losavio, 2014) where some parties struggle to opt in due to a lack of resources, while for others, opting out is impossible. We must not lose sight of how platform urbanism might positively and negatively affect a citizen's quality of life and social participation, through the material practices and idealised discourses that are connected to the platform (Kitchin, 2016).

\section{Conclusion}

Platform urbanism presents a unique opportunity for urban planners, providing a new socio-technical canvas for urban development. The lack of a significant trend in our data indicates the limitations of the smart city concept, and the value of platform urbanism in capturing the liveliness of digitally enabled urbanism. While messiness is analytically not the most pleasant result, it does reflect the lived city more adequately, with its diverse demographics and thus heterogeneity of need and practice. Urban planners should therefore view this as an opportunity to both engage with the messiness, and perhaps create their own 'mess' in the process by experimenting with a localised, platform urbanism that engages more concretely with the situated needs of citizens. Underlying dynamics of interoperability, and associated relations of ( $\mathrm{fr}$ )agility and intermediation, are levers that can be manipulated by planners in their mess-making. This builds on Leszczynski's (2019) observation that platform urbanism is incomplete and prone to malfunctions, yet it is these malfunctions or 'glitches' that allow resistance and innovation to materialise. Such mess-making endeavours by urban planners would serve as a helpful counterbalance to the oligarchical and centralised power structures critics have observed. Although powerful and potentially inequitable, embracing the incoherency and messiness of platform urbanism might be a helpful step in creating new and more vibrant urban spaces.

\section{Acknowledgments}

With great thanks to Sally Tetreault Campbell for providing her editorial support on short notice, and to our research assistant Robert Fleet for his assistance in data collection. We are indebted and extremely grateful to Todd Sanderson, Andreas Duesner, and Andrew Reeson for their intellectual contribution in developing the intellectual frameworks of the broader project. Our 
thanks also go to the broader project team for their contributions and collaborative spirit: Raghav Bhaskar, Xinlong Guan, Guillaume Jourjon, Magnus Moglia, and Thierry Rakotoarivelo. This research is supported by the Science and Industry Endowment Fund (SIEF) at the Commonwealth Science and Industrial Research Organisation, and was undertaken as part of the Data 61 Smart Cities Data Challenge Program, and we are grateful for the support of the fund administrators on this research.

\section{Conflict of Interests}

The authors declare no conflict of interests.

\section{Supplementary Material}

Supplementary material for this article is available online in the format provided by the authors (unedited).

\section{References}

Aoki, P. M., Honicky, R., Mainwaring, A., Myers, C., Paulos, E., Subramanian, S., \& Woodruff, A. (2008). Common sense: Mobile environmental sensing platforms to support community action and citizen science. Paper presented at the Research Showcase @CMU, Carnegie Mellon University, Pittsburgh, USA.

Baldwin, C. Y., \& Woodard, C. J. (2009). The architecture of platforms: A unified view. In A. Gawer (Ed.), Platforms, markets and innovation (pp. 19-44). Cheltenham: Edward Elgar.

Barns, S. (2018). Smart cities and urban data platforms: Designing interfaces for smart governance. City, Culture and Society, 12, 5-12. https://doi.org/10.1016/ j.ccs.2017.09.006

Barns, S. (2019). Negotiating the platform pivot: From participatory digital ecosystems to infrastructures of everyday life. Geography Compass, 13(9), 1-13. https://doi.org/10.1016/gec3.12464

Becker, H. S. (2008). Tricks of the trade: How to think about your research while you're doing it. Chicago, IL: University of Chicago Press.

Bogost, I., \& Montfort, N. (2009). Platform studies: Frequently questioned answers. Paper presented at the Digital Arts and Culture Conference After Media: Embodiment and Context, University of California Irvine, California, USA.

Bosch, J., Olsson, H. H., Björk, J., \& Ljungblad, J. (2013). The early stage software startup development model: A framework for operationalizing lean principles in software startups. Paper presented at the International Conference on Lean Enterprise Software and Systems, Galway, Ireland.

Box, P., \& Lemon, D. (2015). The role of social architecture in information infrastructure: A report for the National Environmental Information Infrastructure (NEII). Canberra: CSIRO.
Boyd, D., \& Crawford, K. (2012). Critical questions for big data: Provocations for a cultural, technological, and scholarly phenomenon. Information, Communication and Society, 15(5), 662-679.

Brannon, M. M. (2017). Datafied and divided: Techno-dimensions of inequality in American cities. City and Community, 16(1), 20-24.

Bronson, K., \& Knezevic, I. (2016). Big Data in food and agriculture. Big Data and Society, 3(1), 1-5. https:// doi.org/10.1177/2053951716648174

Caprotti, F., \& Liu, D. (2020). Emerging platform urbanism in China: Reconfigurations of data, citizenship and materialities. Technological Forecasting and Social Change, 151. Advance online publication. https://doi.org/10.1016/j.techfore.2019.06.016

Cecco, L. (2019, June 6). 'Surveillance capitalism': Critic urges Toronto to abandon smart city project. The Guardian. Retrieved from https://www.theguardian. com/cities/2019/jun/06/toronto-smart-city-googleproject-privacy-concerns

Coletta, C., \& Kitchin, R. (2017). Algorhythmic governance: Regulating the 'heartbeat' of a city using the Internet of Things. Big Data and Society, 4(2), 1-16.

Dohler, M., Mahmoodi, T., Lema, M. A., Condoluci, M., Sardis, F., Antonakoglou, K., \& Aghvami, H. (2017). Internet of skills, where robotics meets $A I, 5 G$ and the Tactile Internet. Paper presented at the European Conference on Networks and Communications (EuCNC), Oula, Finland.

Elmaghraby, A. S., \& Losavio, M. M. (2014). Cyber security challenges in smart cities: Safety, security and privacy. Journal of Advanced Research, 5(4), 491-497.

Gabrys, J. (2014). Programming environments: Environmentality and citizen sensing in the smart city. Environment and Planning D: Society and Space, 32(1), 30-48. https://doi.org/10.1068/d16812

Galdon-Clavell, G. (2013). (Not so) smart cities? The drivers, impact and risks of surveillance-enabled smart environments. Science and Public Policy, 40(6), 717-723. https://doi.org/10.1093/scipol/sct070

Gillespie, T. (2010). The politics of 'platforms.' New Media and Society, 12(3), 347-364. https://doi.org/10. $1177 / 1461444809342738$

Gillespie, M., Osseiran, S., \& Cheesman, M. (2018). Syrian refugees and the digital passage to Europe: Smartphone infrastructures and affordances. Social Media + Society, 4(1), 1-12.

Goggin, G., \& Hjorth, L. (Eds.). (2014). Introduction: Mobile media research-State of the art. London: Routledge.

Hancke, G. P., \& Hancke, G. P., Jr. (2012). The role of advanced sensing in smart cities. Sensors, 13(1), 393-425. https://doi.org/10.3390/s130100393

Harvey, D. (2007). Neoliberalism and the City. Studies in Social Justice, 1(1), 2-13. https://doi.org/10.26522/ ssj.v1i1.977

Helmond, A. (2015). The platformization of the web: Making web data platform ready. Social Media 
+ Society, 1(2), 1-11. https://doi.org/10.1177/ 2056305115603080

Huawei. (2019). Smart city: Leading new ICT, building a better smart city. Huawei. Retrieved from https:// e.huawei.com/au/solutions/industries/smart-city

Hughes, T. P. (1994). Technological momentum. In L. Marx \& M. R. Smith (Eds.), Does technology drive history (pp. 101-114). Cambridge, MA: MIT Press.

Jiang, Q., Kresin, F., Bregt, A. K., Kooistra, L., Pareschi, E., van Putten, E., . . . Wesseling, J. (2016). Citizen sensing for improved urban environmental monitoring. Journal of Sensors, 2016(2), 1-9. https://doi.org/ $10.1155 / 2016 / 5656245$

Kitchin, R. (2014). The real-time city? Big data and smart urbanism. GeoJournal, 79(1), 1-14. https://doi.org/ 10.1007/s10708-013-9516-8

Kitchin, R. (2016). The ethics of smart cities and urban science. Philosophical Transactions of the Royal Society A: Mathematical, Physical and Engineering Sciences, 374(2083), 1-15.

Lambea Llop, N. (2017). A policy approach to the impact of tourist dwellings in condominiums and neighbourhoods in Barcelona. Urban Research and Practice, 10(1), 120-129. https://doi.org/10.1080/17535069. 2017.1250522

Langley, P., \& Leyshon, A. (2017). Platform capitalism: The intermediation and capitalisation of digital economic circulation. Finance and Society, 3(1), 11-31.

Latour, B. (1996). Aramis or the love of technology. Cambridge, MA: Harvard University Press.

Latour, B. (2005). Reassembling the social: An introduction to actor-network-theory. Oxford: Oxford University Press.

Lee, S. U., Zhu, L., \& Jeffery, R. (2018). A contingencybased approach to data governance design for platform ecosystems. Paper presented at the 22nd Pacific Asia Conference on Information Systems (PACIS 2018), Yokohama, Japan.

Leszczynski, A. (2019). Glitchy vignettes of platform urbanism. Environment and Planning D: Society and Space. Advance online publication. https://doi.org/ 10.1177/0263775819878721

McNeill, D. (2015). IBM and the visual formation of smart cities. In S. Marvin, A. Puque-Ayala, \& C. McFarlane (Eds.), Smart urbanism (pp. 34-51). London: Routledge.

Meijer, A., \& Bolívar, M. P. R. (2016). Governing the smart city: A review of the literature on smart urban governance. International Review of Administrative Sciences, 82(2), 392-408. https://doi.org/10.1177\% 2F0020852314564308

Pasquale, F. (2016). Two narratives of platform capitalism. Yale Law and Policy Review, 35(1), 309-319.

Plantin, J.-C., Lagoze, C., Edwards, P. N., \& Sandvig, C. (2018). Infrastructure studies meet platform stud- ies in the age of Google and Facebook. New Media and Society, 20(1), 293-310. https://doi.org/10. 1177\%2F1461444816661553

Schreieck, M., Wiesche, M., \& Krcmar, H. (2016). Design and governance of platform ecosystems: Key concepts and issues for future research. Paper presented at the European Conference on Information Systems (ECIS 2016), Istanbul, Turkey.

Shelton, T., Zook, M., \& Wiig, A. (2015). The 'actually existing smart city.' Cambridge Journal of Regions, Economy and Society, 8(1), 13-25. https://doi.org/ $10.1093 /$ cjres/rsu026

Shilton, K., \& Greene, D. (2019). Linking platforms, practices, and developer ethics: Levers for privacy discourse in mobile application development. Journal of Business Ethics, 155(1), 131-146. https://doi.org/ 10.1007/s10551-017-3504-8

Söderström, O., Paasche, T., \& Klauser, F. (2014). Smart cities as corporate storytelling. City, 18(3), 307-320.

Srnicek, N. (2016). Platform capitalism. Oxford: John Wiley and Sons.

Srnicek, N. (2017). The challenges of platform capitalism: Understanding the logic of a new business model. Juncture, 23(4), 254-257. https://doi.org/10.1111/ newe.12023

Trimi, S., \& Berbegal-Mirabent, J. (2012). Business model innovation in entrepreneurship. International Entrepreneurship and Management Journal, 8(4), 449-465.

van der Graaf, S., \& Ballon, P. (2019). Navigating platform urbanism. Technological Forecasting and Social Change, 142, 364-372. https://doi.org/10.1016/ j.techfore.2018.07.027

van Dijck, J. (2013). The culture of connectivity: A critical history of social media. Oxford: Oxford University Press.

van Dijck, J., \& Poell, T. (2016). Understanding the promises and premises of online health platforms. Big Data and Society, 3(1), 1-11. https://doi.org/10. 1177/2053951716654173

van Dijck, J., Poell, T., \& de Waal, M. (2018). The platform society: Public values in a connective world. Oxford: Oxford University Press.

Wood, D. M., \& Mackinnon, D. (2019). Partial platforms and oligoptic surveillance in the smart city. Surveillance and Society, 17(1/2), 176-182. https://doi.org/ $10.24908 /$ ss.v17i1/2.13116

Zanella, A., Bui, N., Castellani, A., Vangelista, L., \& Zorzi, M. (2014). Internet of things for smart cities. IEEE Internet of Things Journal, 1(1), 22-32. https://doi.org/ 10.1109/JIOT.2014.2306328

Zuboff, S. (2015). Big other: Surveillance capitalism and the prospects of an information civilization. Journal of Information Technology, 30(1), 75-89. https://doi. org/10.1057/jit.2015.5 


\section{About the Authors}
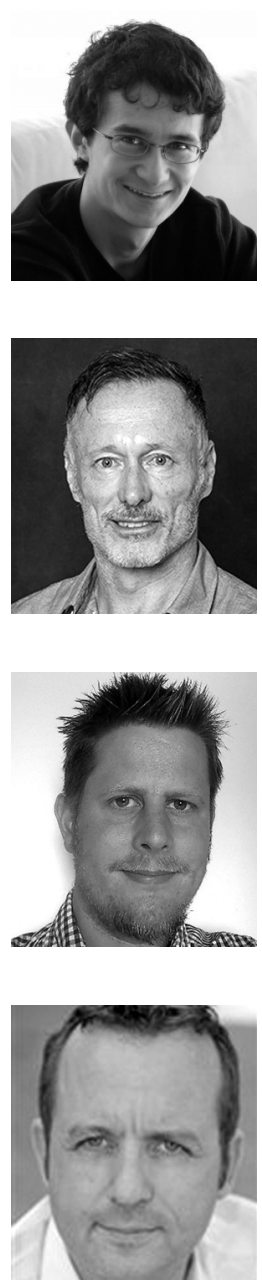

Ashlin Lee (PhD) is a Social Architect at CSIRO and an Honorary Lecturer in Sociology at the Australian National University. With a background in digital sociology, he provides sociological and social science insights to assist in the development, deployment, and optimisation of information and data systems. His work has been published in Information, Communication and Society, Sociology Compass, Surveillance and Society, and the Journal of Sociology. He has worked in academia, public service, user research, and digital transformation roles.

Adrian Mackenzie (PhD) is Professor of Sociology at the Australian National University, and a leading scholar of science and technology studies. His current interests focus on network and computational media, digital sociology and innovation in data-related methods. He is the author of Machine Learners: Archaeology of a Data Practice (2017, MIT Press), and has recently published on the capitalization of online platforms, device-specific events, consumer genomics, and DNA-based biosensing. He also coedits the journal Big Data and Society.

Gavin J. D. Smith (PhD) is Associate Professor of Sociology at the Australian National University. His research looks at the social impacts of digital technologies, data practices and dataveillance across a variety of domains, as well as exploring social relations on the nature-culture borderland. He has published extensively on surveillance practices and politics in journals such as Body and Society, Big Data and Society, The British Journal of Criminology, and Theoretical Criminology. He is the author of Opening the Black Box: The Work of Watching (2015, Routledge), which is based on an ethnography of CCTV camera operators.

Paul Box is a Principle Research Consultant and Social Architect at CSIRO, and leads a team that is researching and designing social, institutional and economic dimensions of Information Infrastructure and platforms; what the team calls Social Architecture. Paul has worked for more than 25 years in geospatial information technology, including with the UN, and NGOs in Asia, Europe, and Africa. He has, for the last 10 years, worked with CSIRO across information infrastructure projects of national significance. 